\title{
Domain of processing and recognition memory for shapes
}

\author{
PAT-ANTHONY FEDERICO \\ Navy Personnel Research and Development Center, San Diego, California 92152
}

\begin{abstract}
An experiment concerning recognition memory for shapes investigated the consequences of manipulating the component operations of domain of processing, that is, encoding strategy, processing time, and stimulus compatibility. It is established that semantic encoding and long processing time facilitated recognition performance for low-, but not for high-, compatibility shapes. A visual task interfered with recognition memory for low-compatibility shapes. Several theoretical contributions to the domain-of-processing framework, which follow from the findings of this experiment, are presented and discussed.
\end{abstract}

The depth-of-processing point of view (Craik \& Lockhart, 1972) posits the existence of a fixed hierarchy or continuum of sequential processing. Stimuli are analyzed initially at a shallow level in terms of their structural or physical features, and subsequently at a deeper level in terms of their semantic or associated attributes. Within this framework, the durability of the memory trace is a direct function of the depth of processing. The processing of a stimulus ceases once the analysis appropriate to the performance of a task is completed. Also, processing time acts as an indicator of depth and, therefore, of the trace's memorability. These initial ideas are unsatisfactory because (1) a postulated series of distinctly different analyses cannot possibly lie on the same continuum, (2) a comprehensive theory must concentrate not only on the nominal stimulus but also on the stimulus in context, and (3) it is very unlikely that the processing of a stimulus stops at the periphery (Baddeley, 1978; Craik \& Tulving, 1975).

Depth-of-processing advocates (Craik \& Tulving, 1975) modified the original concepts to include degree or spread of encoding elaboration. Perceiving individuals can easily elaborate upon the central encoding of a verbal stimulus by further processing it structurally, phonemically, and/or semantically. Contrary to earlier thinking, there is no necessary sequence in which different encoding operations must be executed. These various encoding strategies exist in different dimensions or domains because each analysis is qualitatively distinct from the others. More is involved than a simple metaphor of depth of processing. Spread of encoding elabora-

Opinions or assertions contained herein are those of the author and are not to be construed as official or reflecting the views of the U.S. Department of the Navy. A portion of this research was presented at the meeting of the American Psychological Association, Toronto, Ontario, Canada, August 1978. Requests for reprints should be sent to Pat-Anthony Federico, Navy Personnel Research and Development Center (NPRDC, Code 304), San Diego, California 92152. tion depends on the extent of analysis performed within each processing domain, not on the processing sequence. Within this modified framework, retention is a direct function of the encoding strategy employed. A mere semantic analysis is alleged to be superior to an elaborate structural analysis. Semantic encoding connotes greater stimulus compatibility or congruity with existing cognitive structures, schemata, and rules.

Later, proponents (Lockhart, Craik, \& Jacoby, 1976) maintained that deeper processing refers to two different aspects of encoding. First, among encoding domains, there was a hierarchical organization ranging from shallow, structural analyses to deep, semantic analyses. Second, within encoding domains, stimuli could be further elaborated upon by performing additional processing operations. Therefore, processing typically proceeds through an established sequence of qualitatively distinct analyses; however, not all possible operations are performed within each encoding domain.

Throughout the evolution of depth-of-processing thought there has remained an emphasis on encoding strategy, processing time, and stimulus compatibility as the principal determinants of depth or domain of processing. Hence, to test the suitability of present domain-of-processing speculation, it is necessary to analyze and examine these component processes. Experiments performed to investigate depth of processing typically employ verbal stimuli (e.g., Craik \& Tulving, 1975).

Researchers have conducted two studies of depth of processing and recognition memory using nonverbal stimuli. Bower and Karlin (1974) and Strnad and Mueller (1977) asked subjects to remember pictures of faces while judging them for honesty (deep processing) or sex (shallow processing). Later, recognition performance for deeply processed pictures was superior to those shallowly processed. Neither of these experiments investigated the interactive effects of the proposed components of depth or domain of processing using shape stimuli. Consequently, this deficit unnecessarily 
restricts the generality of current domain-of-processing thinking. To what extent is the present domain-ofprocessing conceptualization applicable to shape stimuli? What are the implications of this theory for recognition memory for shapes?

Within this context, one can hypothesize that semantic encoding, long processing time, or high stimulus compatibility will result in recognition memory for shapes superior to physical encoding, short processing time, or low stimulus compatibility. Further, these domain-ofprocessing components should interact to influence recognition performance as follows. Differential effects of encoding strategy and processing time are possible that depend upon the level of stimulus compatibility. Not being congruent with cognitive structures, shapes of low stimulus compatibility should benefit more from long processing time or semantic encoding than shapes of high stimulus compatibility. The latter stimuli should be more resistant to interfering tasks than should the former.

\section{METHOD}

\section{Subjects and Design}

Subjects were from four companies of Navy recruit trainees who were stationed at the Naval Training Center, San Diego. These recruits had been randomly assigned to their training companies, which consisted of $51,54,55$, and 58 members, respectively. Subjects who did not follow instructions or did not finish the task were discarded. This process, together with randomly discarding other subjects, produced four groups of 46 each. A 2 by 2 by 2 by 2 by 4 univariate factorial design was adopted for the study. There were two between-groups factors: (1) encoding strategy-semantic (SM) or physical (PH), and (2) interference task-verbal (VR) or visual (VZ); and there were three within-groups factors: (1) processing time for encodinglong (LE) or short (SE), (2) stimulus compatibility-low (LC) or high (HC), and (3) number of recognition trials-four. The criterion measures consisted of subjects' confidence ratings in their recognition judgments of $\mathrm{HC}$ or LC random shapes.

\section{Independent-Variables Manipulation}

Encoding strategy was manipulated by instructional set. SM instructions asked the subjects to describe, name, or verbalize some familiar object or situation that the projected random shapes reminded them of. PH instructions asked the subjects to picture, visualize, or imagine the random shapes in their mind's eye. A procedure was followed that forced subjects to comply with the instructional sets: They were required to write a clear SM encoding of each shape or to draw a detailed PH encoding of each shape.

Stimulus compatibility was manipulated as follows. Stimuli were random shapes from a pool scaled according to association value (A) (Vanderplas \& Garvin, 1959), which varied in complexity and the number, content, and heterogeneity of associations. Consequently, eight $\mathrm{HC}$ stimuli were of low complexity (4- and 6-point shapes) and high association value (A $>52$ and $A>54$ ), and eight LC stimuli were of high complexity (24-and 16 -point shapes) and low association value $(\mathrm{A}<28$ and $\mathrm{A}<30)$, respectively. In the Vanderplas and Garvin (1959) identification scheme, the 4- and 6-point shapes are specified as Numbers 1 to 4 and the 24- and 16-point shapes are specified as Numbers 27 to 30 . It was presumed on the basis of Vanderplas and Garvin's data that the sets of 4- and 24-point shapes were equivalent in stimulus compatibility to the sets of 6-and 16-point shapes, respectively.

SE processing time was defined as $.5 \mathrm{sec}$, and LE processing time was defined as $5.0 \mathrm{sec}$. The stimuli consisted of $35-\mathrm{mm}$ slides of black random shapes on transparent backgrounds. These stimuli were displayed by a Kodak Ektagraphic slide projector, Model AF-2, using an f3.5 4- to 6-in. zoom Ektanar lens and a 400-W Sylvania bulb. Encoding processing time or exposure and interstimulus interval were controlled by Hunter decade interval timers, Models 111-B and 111-C, respectively. These were interconnected with the projector and a Mackenzie Adjusto-Dissolve, Model AD-2. A Wallmaster Radient daylight screen was positioned in front of a room of sufficient size to accommodate one of the four groups at a time. The projector to screen distance was $24.5 \mathrm{ft}$. The projected stimulus shapes were approximately $6 \mathrm{ft}$ wide and $4 \mathrm{ft}$ high. Ambient illumination within the room was $3.0 \mathrm{fc}$.

\section{Dependent-Variable Measurement}

Recognition memory for random shapes was defined as follows. Subjects were shown HC and LC random shapes. After having seen each of these subsets of stimuli, they were required to select those shapes they had seen before from among similar shapes in two larger sets that included some they had not seen before. As each stimulus was projected during recognition testing, subjects were asked to rate their confidence in their recognition judgments. They checked on a response form either "seen before" or "not seen before" and 20\%, $40 \%, 60 \%, 80 \%$, and $100 \%$ to indicate their degree of confidence in their decisions. These response categories (see before, not seen before, and $20 \%, 40 \%, 60 \%, 80 \%$, and $100 \%$ ) were scored plus or minus and $1,2,3,4$, or 5 , respectively.

\section{Procedure}

From each cluster of four 4-, 6-, 16-, and 24-point shapes, two were randomly selected to be target stimuli. The remaining two shapes within each cluster served as distractor stimuli during recognition testing. Target shapes were displayed for either LE or SE processing times. Within each processing time, subjects were presented HC and LC shapes. The presentation sequence of LE-SE processing time was fixed across the encoding groups. Similarly, the presentation sequence of LC-HC shapes was fixed across processing times.

When all subjects understood the instructions, four 8-point random shapes were displayed for practice purposes at the SE and LE processing times, with an interstimulus interval of $10 \mathrm{sec}$. Afterward, eight target stimuli, consisting of four HC shapes and four LC shapes, were shown for a SE or a LE processing time, using an interstimulus interval of $20 \mathrm{sec}$. Within this interval, subjects were to write or to draw their encoding for each stimulus in their response booklet. The subjects were then instructed to turn to the next blank page in the booklet to ensure that the encodings were synchronized with the proper target stimulus. Following the encoding of these four $\mathrm{HC}$ and LC shapes, subjects were given an interference task.

One of the SM or PH encoding groups completed a VR interference task while the other encoding groups completed a VZ interference task. The VR interference activity consisted of taking both parts of the Extended Range Vocabulary Test (V-3) and the Advanced Vocabulary Tests (V-4 and V-5), and the VZ interference activity consisted of taking both parts of the Form Board Test (Vz-1) and the Surface Development Test (Vz-3) from the Kit of Reference Tests for Cognitive Factors (French, Ekstrom, \& Price, Note 1). The total time to complete the VR and VZ interference task was $28 \mathrm{~min}$.

After the subjects finished the interference activity, they were given the recognition test. Sixteen test stimuli consisting of four 24-, 4-, 16-, and 6-point shapes, each set of which included two target and two distractor shapes, were displayed using an exposure of $2.75 \mathrm{sec}$ with an interstimulus interval of $10 \mathrm{sec}$. During each $10 \mathrm{-sec}$ interval, the subjects responded to the preceding test stimulus by indicating in the booklet the amount of confidence in their recognition judgments. Subsequently, the subjects were given 5 min to complete the openended postexperimental questionnaire. 


\section{RESULTS}

A 2 by 2 by 2 by 2 by 4 univariate factorial analysis of variance was computed on the subjects' scored confidence ratings. The main effects of processing time and stimulus compatibility were significant at the .001 level $[\mathrm{F}(1,183)=50.33$, $\mathrm{MSe}=8.42$, and $\mathrm{F}(1,183)$ $=38.65$, MSe $=12.30$, respectively $]$. The mean confidence ratings for target stimuli, hit rate, and recognition capability $\left(d^{\prime}\right)$ for the main effect of processing time were $3.65,87.36$, and 2.12 for LE, and 2.53, 75.68, and 1.85 for SE, respectively. For the main effect of stimulus compatibility, these statistics were 2.06, 70.92 , and 1.56 for LC, and $4.12,92.12$, and 2.58 for $\mathrm{HC}$, respectively. For target stimuli, which were initially encoded, LE processing time led to better recognition performance than did SE processing time. This was reversed for distractor stimuli. Overall recognition performance for HC shapes exceeded that of LC shapes.

The interaction of encoding strategy and stimulus compatibility was significant at the .001 level $[\mathrm{F}(1,180)$ $=20.80, \mathrm{MSe}=10.53 \mathrm{]}$. The Tukey technique indicated that there was a differential effect of encoding strategy that depended upon the specific level of stimulus compatibility. The mean confidence ratings for target stimuli, hit rate, and recognition capability were 2.77 , 89.40 , and 2.68 for SM-LC, 4.22, 96.33, and 3.50 for SM-HC, 1.36, 81.52, and 2.29 for PH-LC, and 4.03, 95.79, and 3.22 for PH-HC, respectively. Within LC target stimuli, there was a difference in recognition performance between SM and $\mathrm{PH}$ encoding strategies; however, within HC target stimuli, there was none. Recognition performance was superior for semantically encoding LC shapes compared with physically encoding them. Performance was the same whether HC shapes were encoded semantically or physically. For both encoding strategies, recognition performance for $\mathrm{HC}$ stimuli exceeded that for LC stimuli.

The interaction of processing time and stimulus compatibility was significant at the .001 level $[F(1,183)$ $=16.42, \mathrm{MSe}=9.65]$. The Tukey procedure revealed that the impact of processing time was contingent upon the degree of stimulus compatibility. The mean confidence ratings for target stimuli, hit rate, and recognition capability were $2.99,90.62$, and 2.62 for LE-LC, $4.31,96.74$, and 3.35 for LE-HC, 1.13, 80.30, and 2.42 for SE-LC, and 3.94, 95.38, and 3.19 for SE-HC, respectively. Within LC target stimuli, there was a difference in recognition performance between LE and SE processing times, yet within $\mathrm{HC}$ target stimuli there was none. Recognition performance was superior for processing LC shapes over a long compared with a short period. Performance was the same for HC shapes over both processing intervals. For the two processing times, recognition performance for $\mathrm{HC}$ stimuli bettered that for LC stimuli.

Finally, the interaction of interference task and stimulus compatibility was significant at the .01 level
$[F(1,180)=10.52, \mathrm{MSe}=10.52]$. The Tukey technique indicated that there was a differential effect of interference task that was a function of stimulus compatibility. The mean confidence ratings for target stimuli, hit rate, and recognition capability were $1.49,82.34$, and 2.33 for VZ-LC, 4.09, 95.92, and 3.22 for VZ-HC, $2.64,88.59$, and 2.63 for VR-LC, and $4.17,96.20$, and 3.50 for VR-HC, respectively. Within LC target stimuli, there was a difference in recognition performance as indexed by confidence ratings between VZ and VR interference tasks, whereas within $\mathrm{HC}$ target stimuli there was none. The visual interference task inhibited recognition memory for $L C$ shapes more so than the verbal interference task for these stimuli. However, no differential effect was established for these two interference tasks for HC shapes. For both interference tasks, recognition performance for $\mathrm{HC}$ stimuli as assessed by confidence ratings surpassed that for LC stimuli.

A content analysis of the codes generated by the subjects in the SM encoding group to facilitate their recognition memory was conducted. Considerably more of them used three or more words to mediate their responses to LC stimuli than to $\mathrm{HC}$ stimuli. These subjects seemed to take into consideration the details, irregularity, and bizarreness of LC shapes. In addition, there was more homogeneity among the subjects' encodings and less differentiation of qualification by the use of adjectives or adverbs for HC shapes than for LC shapes. An analysis of the codes produced by the subjects in the $\mathrm{PH}$ encoding group indicated that $\mathrm{HC}$ stimuli were reproduced with more accuracy than were LC stimuli. Apparently, the high complexity of the LC shapes relative to the $\mathrm{HC}$ shapes interfered with the quality of the subjects' pictorial encoding. The posttest experimental questionnaires revealed that the subjects in both encoding groups had no difficulty in consistently following instructions. However, several indicated that they had trouble producing encoding for LC shapes.

\section{DISCUSSION}

In light of the results, it is not completely correct to argue, as it has been argued within the depth-of-processing framework (Craik \& Lockhart, 1972; Craik \& Tulving, 1975; Lockhart et al., 1976), that long processing time or SM encoding produces better recognition performance than short processing time or PH encoding. This must be qualified and modified as follows. Some of the findings indicated that the effects of processing time and encoding strategy are contingent upon the level of stimulus compatibility. Long processing time or SM encoding facilitated recognition performance primarily for LC shapes. It did not make any difference how HC shapes were processedeither for long or short periods, or with $\mathrm{PH}$ or SM encoding strategies-recognition performance for these stimuli was not affected.

HC shapes by their very nature are congruent with prevailing cognitive structures. Because of their simple contours, these stimuli can be rapidly analyzed, easily encoded, and accurately recognized. Consequently, any additional processing of these HC shapes in terms of longer encoding times or by means of enrichment or elaboration will be unproductive, due to a possible ceiling effect. Additional processing need not be resorted to 
for these stimuli, which can be easily stored as simple structural traces. Physically encoding them can efficiently serve as the basis of their distinctiveness and recognizability.

LC shapes do not easily allow physical traces of their contours to be stored and recognized because of their inherent complexity. For these stimuli, additional processing by means of SM encoding or longer acquisition times not only provided stable, readily made representations, but also encouraged a visual search for critical or distinctive features. Further processing of LC stimuli distinguished these shapes and therefore enhanced their recognition by possibly minimizing confusion among them.

Depth of processing was considered as qualitatively distinct domains of encoding rather than a continuum of processing. Therefore, it was not necessary to employ an independent index to specify depth. Obviously, processing time per se is not a suitable indicator of depth. Tasks that demand PH encoding can take a long time because of the amount of processing they require, or familiar stimuli can be rapidly processed to a semantic level. Nevertheless, the depth-of-processing model assumes that within a specific type of stimuli, for example, within compatibility levels, deeper processing demands more time (Craik \& Tulving, 1975). As found, long, not short, processing time produced superior recognition performance for LC shapes. These specific results partially support the existence of a continuum of processing.

Other findings demonstrated that the consequences of modality-specific interfering tasks were also contingent upon the level of stimulus compatibility. Distinct visual and verbal inhibitory activities had no differential effect upon recognition memory for HC shapes. Yet, recognition performance for LC shapes was inhibited more by the visual than the verbal interfering task. This difference suggested that the visual representational system was greatly taxed in processing LC stimuli and storing their physical traces for subsequent recognition. Possible interference for $\mathrm{HC}$ stimuli was likely eliminated or nullified by the inherently simple contours and intrinsic meaningfulness of these shapes. HC stimuli easily lend themselves to processing at a semantic level in addition to their processing at a physical level. Hence, interference that was produced at a physical level could have been readily curtailed by also processing these shapes at a semantic level (Nelson, Wheeler, \& Brooks, 1976).

Several theoretical contributions to the domain-of-processing framework follow from the findings of this experiment. Recognition performance does not exclusively depend upon elaboration or enrichment of encoding. Nevertheless, an interaction exists between the encoding strategies employed and the compatibilities of the stimuli with prevailing cognitive structures. Contrary to the revised depth-of-processing model, the proposed beneficial effect of SM encoding does not occur with stimuli of high compatibility. This differential effect appears only with stimuli of low compatibility. Recognition memory for these shapes is enhanced by semantically encoding them. Stimuli that are enriched by associations result in more elaborate encodings and longer lasting traces. This enrichment could act to integrate these LC stimuli with existing cognitive structures at the time of encoding. Use can be made of accumulated experiences and acquired schemata to semantically encode these shapes. This, in turn, could facilitate the storage and retrieval of the traces of these stimuli during recognition.
Presenting a LC shape for subsequent recognition testing could easily redintegrate the complete semantic structure that serves as a context while encoding takes place. Consequently, recognition memory for LC stimuli is improved by SM encoding because (1) more elaborate traces are initially established, and (2) more enriched encodings suggest greater congruity with prevalent cognitive structures. It is these schemata that are elicited to facilitate the retrieving and identifying processes during later recognition. This theoretical interpretation could easily explain the results obtained by others (Bower \& Karlin, 1974; Strnad \& Mueller, 1977). Superior recognition itself is contingent upon accurately retrieving or reconstructing the semantic representations of the encoded stimuli. Traces such as these are likely to be unique and distinctive by referring to discriminating features that in turn facilitate recognition performance (Lockhart et al., 1976). No claim is made that the process of recognition involves a match with a stored trace. Yet, SM encoding of LC stimuli makes another type of information available, in addition to physical properties, that can be used to better the recognition process.

\section{REFERENCE NOTE}

1. French, J. W., Ekstrom, R. B., \& Price, L. A. Manual for kit of reference tests for cognitive factors. Princeton, N.J: Educational Testing Service, 1963.

\section{REFERENCES}

BADDEley, A. D. The trouble with levels: A reexamination of Craik and Lockhart's framework for memory research. Psychological Review, 1978, 85, 139-152.

Bower, G. H., \& Karlin, M. B. Depth of processing pictures of faces and recognition memory. Journal of Experimental Psychology, 1974, 103, 751-757.

Craik, F. I. M., \& Lockhart, R. S. Levels of processing: A framework for memory research. Journal of Verbal Learning and Verbal Behavior, 1972, 11, 671-684.

Craik, F. I. M., \& Tulving, E. Depth of processing and the retention of words in episodic memory. Journal of Experimental Psychology: General, 1975, 104, 268-294.

French, J. W., Eкstrom, R. B., \& Price, L. A. Manual for kit retention of words in episodic memory. Journal of Experimental Psychology: General, 1975, 104, 268-294.

Lockhart, R. S., Craik, F. I. M., \& Jacoby, L. Depth of processing, recognition and recall. In J. Brown (Ed.), Recall and recognition. New York: Wiley, 1976.

Nelson, D. L., Wheeler, J. W., \& Brooks, D. H. Meaning and the elimination of sensory interference. Journal of Experimental Psychology: Human Learning and Memory, 1976, 2, 95-102.

Strnad, B. N., \& Mueller, J. H. Levels of processing in facial recognition memory. Bulletin of the Psychonomic Society, $1977,9,17-18$.

Vanderplas, J. M., \& Garvin, E. A. The association value of random shapes. Journal of Experimental Psychology, 1959, 57, 147-154.

(Received for publication July 28, 1980.) 